\title{
A study of 3D Strike-Zone Design and Its Track Based on Kinect v2
}

\author{
Ching-Tang Hsieh ${ }^{1,}$, , Li-Ming Chen ${ }^{1, b}$, Ku-Chen Huang ${ }^{2}$, Meng-Shiuan Pan ${ }^{3}$ \\ and Hsing-Che Liou $^{1}$ \\ ${ }^{1}$ Dept. of Electrical Engineering, Tamkang University, 25137, New Taipei, Taiwan \\ ${ }^{2}$ Office of Physical Education , Tamkang University, 25137, New Taipei, Taiwan \\ ${ }^{3}$ Dept. of Information Engineering, Tamkang University, 25137, New Taipei, Taiwan \\ ahsieh@ee.tku.edu.tw, ${ }^{b} \mathrm{~ms} 0071799 @$ hotmail.com
}

Keywords: Kinect, 3D K-Zone, Baseball.

Abstract. An increasing number of researchers have begun to use depth sensors in their studies due to the drop in sensor prices in recent years. In computer vision, depth provides a wider range of useful information. In sports, baseball and softball pitchers are typically unable to self-evaluate their throw quality when practicing in the bullpen, and umpires are mostly unavailable to evaluate their throw quality. In this context, the present study proposed a 3D strike zone system based on the Kinect v2. The system was able to determine ball speed and throw quality. Recordings were then exported into a visual ball trajectory image including a 3D K-Zone, which can service as a reference for pitchers.

\section{Introduction}

Baseball is a popular sport and major competitions are frequently held, such as league competitions. In a baseball game, the performance of the pitcher is vital to the outcome of the game. When training in the bullpen, pitchers are largely unable to analyze the trajectories of their throws themselves and require an observer to determine the quality of their throws.

The Hawk-Eye System [1] [2] computerized ball tracking system was introduced by Roke Manor Research Limited in 2001. It is now widely employed in various sports, such as cricket and tennis. The system tracks ball trajectories, displays a record as a moving image, and predicts the most likely ball paths. The system is typically used in ball games to determine ball trajectories, reducing the occurrence of misinterpretations from eye tracking due to fast ball speeds. Thus, this system is now an integral part of the determination process. The system uses a varying number of cameras for different ball games. The configuration for a tennis match uses ten high-speed cameras (Figure 1a) to track the ball trajectories at different angles and convert the data using a computer to output a 3D image (Figure 1b). A large display is then used to display the ball paths and landings. The conversion process requires less than ten seconds. This system is widely used in televised ball games. However, this technology is extremely expensive, and decisions are still based on the judgments of the umpire. The system is only used as a supplementary means for judgments.

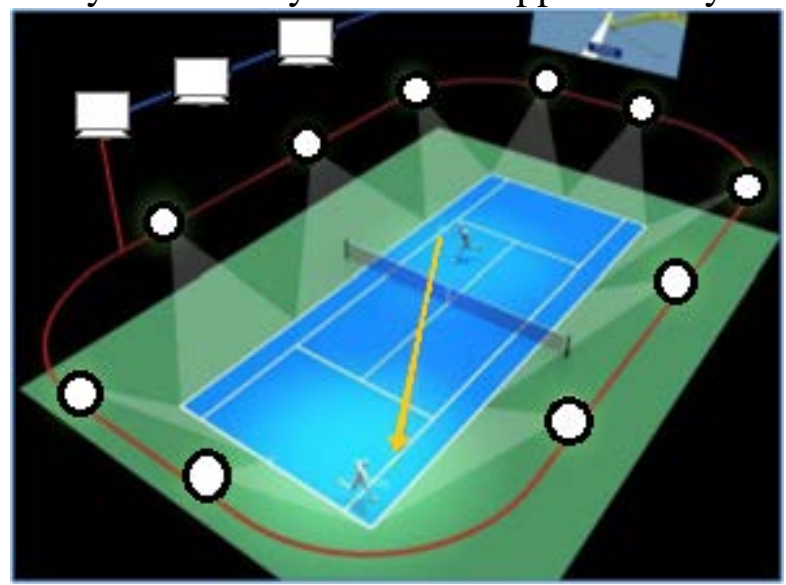

(a)

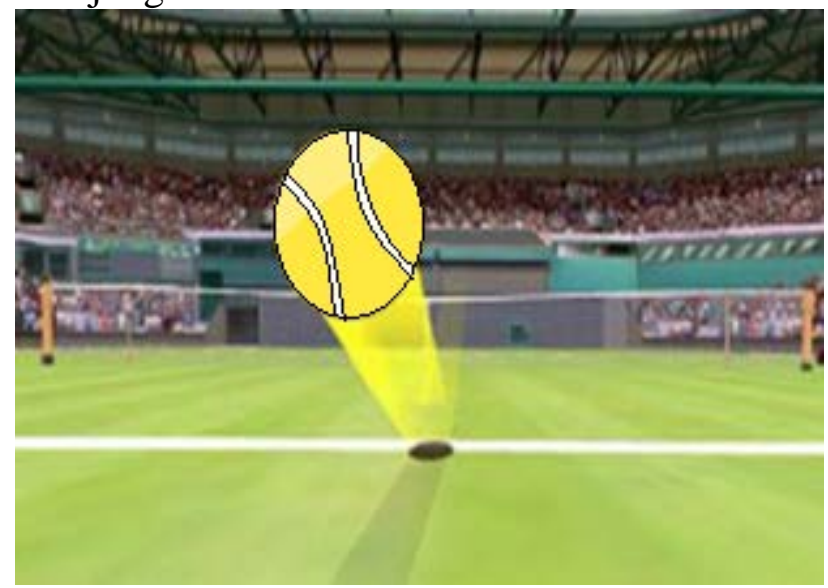

(b)

Figure 1. The system of Hawk Eye [1] 


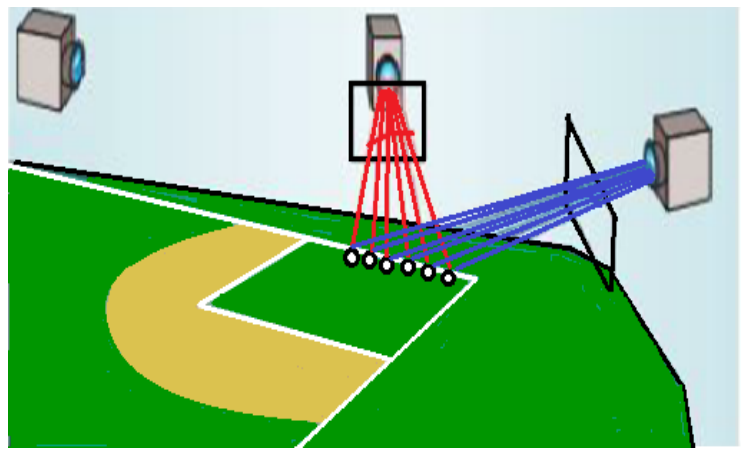

Figure 2. The cameras setting of Traditional K-Zone

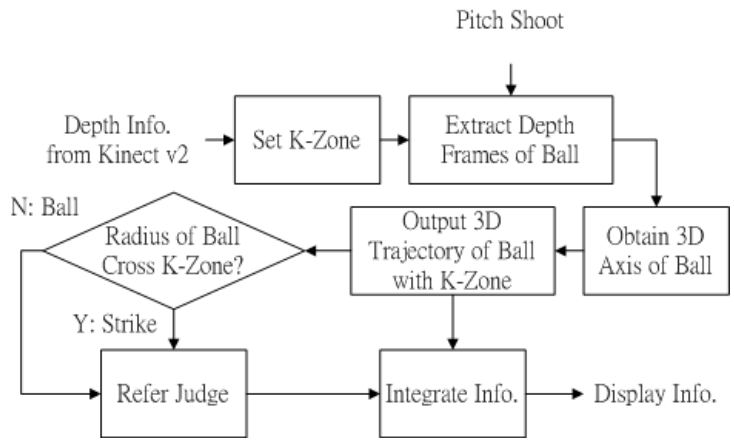

Figure 3. System flow chart we proposed

In the present study, we used a Kinect v2 sensor to create a ball tracking system. A 3D K-Zone is established at the home plate to collect depth data concerning the ball trajectory. This information is used to position the ball in a 3D space and determine the quality of the throw. A graphics software was then employed to illustrate the trajectories in the 3D space onto a 3D image. This image can be provided as a reference to pitchers to self-evaluate and self-adjust their throws.

\section{K-Zone}

The Entertainment Sports Programming Network (ESPN) and Sport-Vision introduced K-Zone [3] in 2001 to judge throw quality. Since then, reliance on this technology has increased exponentially. K-Zone has two primary functions, namely, to facilitate the judgment of throw quality and to illustrate ball trajectories. The purpose of these functions is to represent more clearly the ball trajectories for audiences watching baseball games on TV.

Traditional K-Zone systems comprise of three cameras [2], one positioned behind the pitcher, one behind the batter, and one perpendicular to the pitcher and the batter. The three-camera configuration is illustrated in Figure 2. Numerous previous studies have based their research on this configuration [4-10]. Images are extracted from the recordings to determine the strike zone at the location of the batter and record the positions of various throws. The positions of the throws are linked to form ball trajectories and used to determine throw quality, type, and speed.

\section{Proposed Method}

The present study developed a system that uses the depth data collected by a Kinect v2 to analyze ball trajectory and relevant information. The system procedures are illustrated in Figure 3.

First, the Kinect v2 was set up above the home plate. The depth images captured by the Kinect v2 were processed using the Kinect SDK 2.0 to correct image color and depth data [11]. Figure 4 illustrates the K-Zone established using the positions of the depth data. Once the K-Zone was established, the pitching frames of the pitcher were captured. The frames were processed using background reduction to obtain the ball positions, as shown in Figure 5. The closed operation of morphology was adopted to remove the noise from the depth data. The ball positions were scanned clockwise from the ball, individually processing every frame. When a non-zero pixel is scanned, the center is moved to the non-zero pixel, the pixel is deleted, and the center moves back to the center of the ball. This process is repeated to illustrate the center binary coordinates and depth data, which is combined with the K-Zone data to illustrate the ball trajectory.

Once the ball trajectory is established, the distance between the pixels contained in the trajectory is calculated. The trajectory is also analyzed to ensure that it is not smaller than the radius of the ball, and the throw quality is determined. The data were collated to form a rotatable 3D ball trajectory. The aforementioned reference quality information was collated and exported by the system. This information can then be provided to users for self-evaluation, self-adjustment, and optimization. 


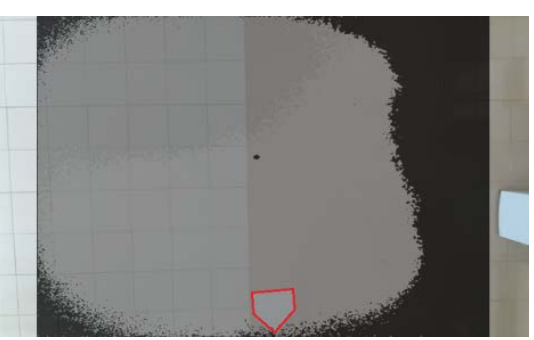

Figure 4. Correct color and depth information by SDK

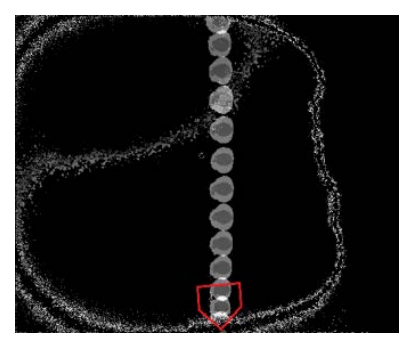

Figure 5. Ball position extracted from frames

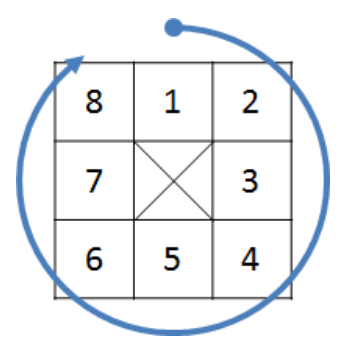

Figure 6. Colck-wise Erosion Scan

\section{Experimental Result}

The equipment used in the present study was as follows: (1) a laptop equipped with an Intel ${ }^{\circledR}$ CoreTM i7 4700HQ CPU processor; (2) Windows 8.1 operating system; (3) Kinect v2 sensor; and (4) color cameras set at an image resolution of $1920 \times 1080$ and a depth resolution of $512 \times 484$. The cameras were able to measure depth of 500 to $4500 \mathrm{~mm}$ at $30 \mathrm{fps}$. Microsoft Visual Studio 2013 and OpenCV ver. 2.4.11 were adopted as the control software linked to the Kinect v2 sensor. The software was used to process the captured images and output reference judgments. Moreover, html and JavaScript were used in the present study to illustrate the 3D data into ball trajectories and the 3D strike zone.

For the proposed system, the sensor was installed four meters above the home plate to capture the depth data of the plate and the surrounding area. Figure 7 shows the front and side data captured by the sensor and Figure 8 shows the ball trajectory and 3D strike zone.

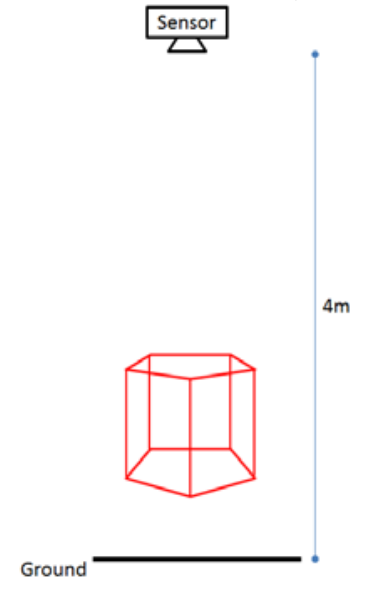

(a) Façade View

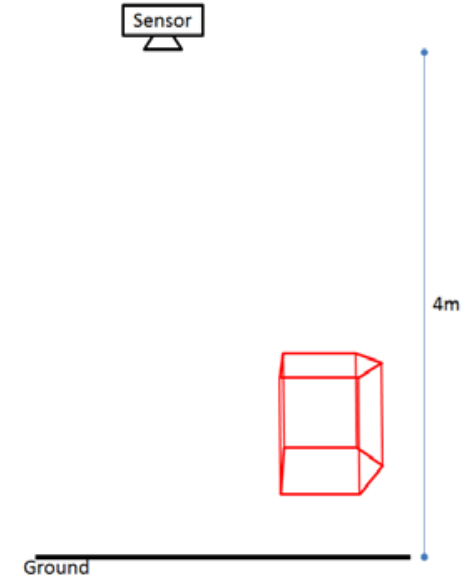

(b) Side View

Fig.7 Experimental Environment Setting

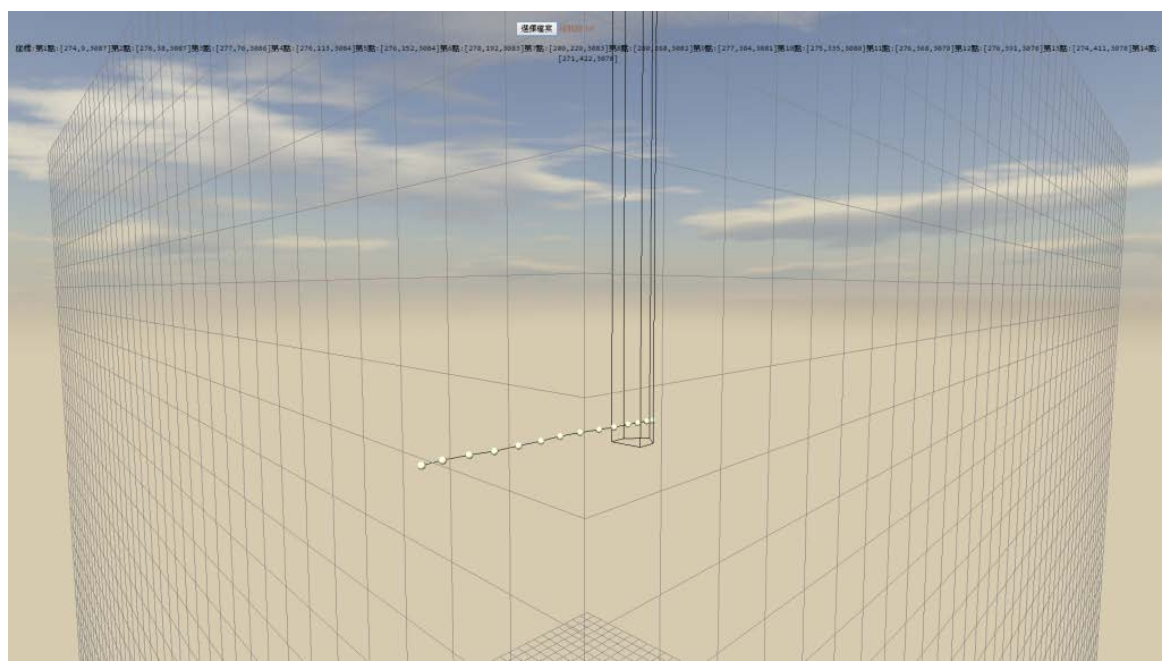

Fig.8 Shooting Trajectory with 3D K-Zone 


\section{Conclusion and Future Work}

In the past, systems that were able to measure the K-Zone were extremely expensive and were only used to provide references to support umpire judgments. These systems could not be standardized and applied to training locations. The present study proposed a system that uses the Kinect v2 sensor to provide pitchers with 3D K-Zone information when they train alone. The information provided by the system can help users self-adjust their ball trajectories, allowing them optimize their conditions during training or warm up.

\section{Acknowledgements}

This work was financially supported by the Tamkang University under grant number FDRX10-2321.

\section{References}

[1] International Bureau of Education (IBE) "Video Processor Systems for Ball Tracking in Ball Games.” International Application Published Under the Patent Cooperation Treaty, WO 01/41884 A1, June 2001.

[2] N. Owens, C. Harris, and C. Stennett. "Hawk-eye tennis system,” IEEE International Conference on Visual Information Engineering, pp.182-185, 2003.

[3] A. Gueziec, et al., "Tracking Pitches for Broadcast Television," Proc. in IEEE International Conference on Computer, Vol. 35, No. 3, pp.38-43, Mar. 2002.

[4] W. T. Chu, C. W. Wang, and J. L. Wu, "Extraction of baseball trajectory and physics based validation for single view baseball video sequences," IEEE International Conference on Multimedia \& Expo, pp. 1813 1816, 2006.

[5] H. S. Chen, H. T. Chen, W. J. Tsai, S. Y. Lee and J. Y. Yu, "Pitch-by-pitch Extraction from Single View Baseball Video Sequences", IEEE International Conference on Multimedia and Expo, pp. 1423-1426, 2007.

[6] H. T. Chen, H. S. Chen, M. H. Hsiao, W. J. Tsai, and S. Y. Lee, “A trajectory based ball tracking framework with visual enrichment for broadcast baseball videos,” Journal of Information Science and Engineering, Vol. 24, pp. 143-157, 2008.

[7] C. F. Lee. "Study on Batter-dependent Strike Zone Identification from TV broadcast Images." National Taiwan University of Science and Technology, Dept. of Electrical Engineering, Master Thesis, 2008. (Written by Traditional Chinese.)

[8] W. Z. Ma. "Baseball detection and tracing system by multiple cameras." National Taiwan University of Science and Technology, Dept. of Electrical Engineering, Master Thesis, 2008. (Written by Traditional Chinese.)

[9] Y. W. Fu. "Baseball Pitch Recognition Using Hierarchical Boosting Algorithm.” National Central University, Dept. of Communication Engineering, Master Thesis, 2009. (Written by Traditional Chinese.)

[10] C. Y. Wang. "Reconstruction of 3D baseball trajectories under the Cloud service." Hsuan Chuang University, Dept. of Information Management, Master Thesis, 2013. (Written by Traditional Chinese.)

[11] Microsoft “Kinect for Windows SDK.” Microsoft MSDN Library Developer Network. https://msdn.microsoft.com/en-us/library/dn799271.aspx. 